\title{
The Bound $\mathrm{Na}^{+}$is Negative Effecter for Thrombin-Substrate Stereospecific Complex Formation
}

\author{
Ikuo Kurisaki ${ }^{\dagger \dagger}$, Masayoshi Takayanagi ${ }^{\dagger, \ddagger}$, Masataka Nagaoka ${ }^{*} \dagger, \dagger$ \\ ${ }^{\dagger}$ Graduate School of Information Science, Nagoya University, Furo-cho, Chikusa-ku, Nagoya 464-8601, \\ Japan \\ ॠCore Research for Evolutional Science and Technology, Japan Science and Technology Agency,
}

Honmachi, Kawaguchi 332-0012, Japan

\footnotetext{
* Corresponding author
}

Professor Masataka Nagaoka; Email: mnagaoka@is.nagoya-u.ac.jp; phone and fax: +81-52-789-5623. 


\section{SI-1. MD simulations of thrombin-substrate complex}

\section{System setup}

We prepared atomic coordinates of thrombin-chromogenic substrate (Phe-Pro-Arg-p-nitroanilide: FPR)-Na ${ }^{+}$complex structure using thrombin-PPACK complex structure (PDB entry: 1PPB) ${ }^{1}$. The PPACK was converted into FPR by in silico modeling. In biochemical experiments, a D enantiomer of Phe is used at the $\mathrm{N}$-terminal of FPR. ${ }^{2}$ However, we used a $\mathrm{L}$ enantiomer of Phe in our simulations, keeping in mind thrombin's interaction with a natural substrate. All crystal water molecules were removed from the original PDB structure. $\mathrm{N} \delta$ and $\mathrm{N} \varepsilon$ protonation states are employed for His57 in the catalytic triad and the other histidine residues, respectively. Furthermore, the four disulfide bonds in thrombin were formed. ${ }^{1}$

This thrombin-FPR complex structure was superposed on a thrombin- $\mathrm{Na}^{+}$complex structure (PDB entry 1SG8) ${ }^{3}$ using RMS-fit with UCSF Chimera ${ }^{4}$. After the superposition of these two structures, the atomic coordinates of the thrombin- $\mathrm{Na}^{+}$ complex were removed but those of the bound $\mathrm{Na}^{+}$and 6 crystal water molecules in the $\mathrm{Na}^{+}$-binding region. This was used to prepare thrombin-FPR-Na ${ }^{+}$system. The thrombin-FPR complex structure was also superposed on a $\mathrm{Na}^{+}$-free thrombin structure (PDB entry 1 SGI) to add the 4 crystal water molecules in the $\mathrm{Na}^{+}$-binding region. ${ }^{3}$ This 
was used to prepare thrombin-FPR system.

Each of the thrombin-FPR and thrombin-FPR-Na ${ }^{+}$complex structures was solvated in the rectangle box, which was set so that the minimum distance from the box's face to the thrombin surface was $10 \AA$. Finally, 11547 water molecules were added to each system. Each of solvated structures were electronically neutralized to simulate $140 \mathrm{mM}$ $\mathrm{NaCl}$ by adding $\mathrm{Na}^{+}$and $\mathrm{Cl}^{-}$molecules $\left(33 \mathrm{Na}^{+}\right.$and $37 \mathrm{Cl}^{-}$for thrombin-FPR; $32 \mathrm{Na}^{+}$ and $37 \mathrm{Cl}^{-}$for thrombin-FPR-Na+ ${ }^{+}$. The atomic coordinates obtained above was used as an initial structure of the following simulations. Molecular modelings were performed using the LEaP module in AmberTools 1.5 package. $^{5}$

\section{Force field parameter sets}

To calculate the forces acting among atoms, AMBER force field $99 \mathrm{SB}^{6}, \mathrm{SPC} / \mathrm{E}$ water $\operatorname{model}^{7,8}$, and JC ion parameters adjusted for the SPC/E water model ${ }^{9,10}$ were applied for amino acid residues, water molecules, and ions, respectively. A set of force field parameters for FPR are prepared by using quantum mechanical calculation (see the following for details). Simulations for the solvated systems were performed under the periodic boundary condition. Electrostatic interaction was treated by the Particle Mesh Ewald method, where the real space cutoff was set to 9 [^]. The vibrational motions 
associated with hydrogen atoms were frozen by the SHAKE algorithm.

\section{Prepare force field parameters for residues in FPR}

We prepared force field parameters for unusual residues in FPR, namely, deprotonated N-terminal phenyl alanine (DNF) and para-nitroaniline (PNA), which are located at the N- and C-terminals of FPR, respectively. Considering the earlier study on capped polypeptides ${ }^{11}$, we assumed that the $\mathrm{N}$-terminal amid group of the FPR molecule is deprotonated. The molecular structures of DNF and PNA were prepared using Facio ${ }^{12}$ (Figures S1 and S2). In the quantum mechanical calculations, DNF and PNA were capped by NME at the C-terminal and $\mathrm{ACE}$ at the N-terminal, respectively. Each molecular structure was energetically minimized at the Hartree-Fock/6-31G* level of calculation by using Gaussian $03^{13}$ and the RESP charges were calculated for each atom in the residues. The sets of atomic charges of DNF and PNA are shown in Tables S1 and S2, respectively. The other force field parameters (bond, angle, dihedral and van der Waals parameters) were assigned using the general Amber force field (GAFF) 1.4 parameters $^{14,15}$.

\section{Molecular dynamics simulations}


Using each of the solvated thrombin-FPR and thrombin-FPR-Na ${ }^{+}$complex structures, we prepared the independent 100 initial atomic coordinates for each system by randomizing positions of ion molecules. The ion-randomization procedure consists of 4 steps: (1) randomization of ion's atomic coordinates; (2) energetic relaxation of solvent molecules; (3) second randomization of ion's atomic coordinates; (4) second energetic relaxation of solvent molecules. In the first and third steps, the cation bound to thrombin is excluded in randomization of ions. In the second and fourth steps, thrombin and the bound cation were restrained around the initial structure. The detail of this four-step procedure was already reported in our earlier study ${ }^{16}$. For each system, we repeated this procedure by 100 times and obtained independent 100 initial atomic coordinates.

For each system, using each of 100 initial atomic coordinates obtained above, the following 6-step MD simulations were performed: NVT (1 to 300 [K], 30 [ps], 10

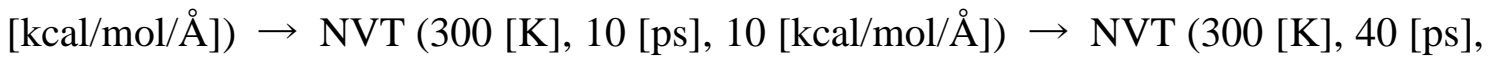

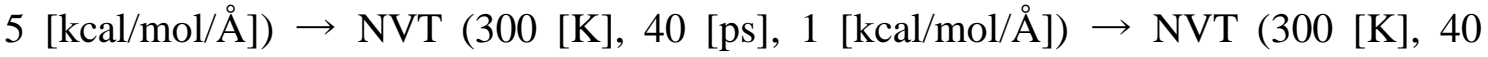
[ps]) $\rightarrow$ NPT (300 [K], 1 [bar], 25 [ns]). The last 25 ns NPT simulation was used for the following analyses. Each simulation employed the time step of 2 fs for the integration. In the first NVT simulation, the reference temperature was linearly increased along the time course. In the first 4 simulations, thrombin and the bound $\mathrm{Na}^{+}$ 
were restrained by harmonic potential referring the initial atomic coordinates for each of the simulations. In each NVT simulation, temperature was regulated using Langevin thermostat with $1 \mathrm{ps}^{-1}$ collision coefficient. In the last $25 \mathrm{~ns}$ NPT simulation, temperature and pressure are regulated by Berendsen thermostat ${ }^{17}$ with a 5 ps coupling constant and Berendsen barostat ${ }^{17}$ with a 0.1 ps coupling constant, respectively. The initial atomic velocities were randomly assigned from the Maxwellian distribution at 1

$\mathrm{K}$. The translational center-of-mass motion was removed by every 500 steps to keep the whole system around the origin, avoiding the overflow of coordinate information from the MD trajectory format.

Randomization of ion's atomic coordinates and both MD and MM simulations were performed using ptraj module in AmberTools 1.5 package ${ }^{5}$ and CPU-version PMEMD module in AMBER 12 package $^{18}$, respectively. The sixth step MD simulation procedure, i.e., the last 25 ns NPT simulation, was performed by GPU-version PMEMD module based on SPFP algorism ${ }^{19}$ with NVIDIA GeForce GTX680.

\section{Analyses of MD trajectories}

Using the ptraj module in AmberTools 1.5 package ${ }^{5}$, we calculated RMSD to X-ray crystallographic structure of thrombin (PDB entry: $1 \mathrm{PPB})^{1}$ as for the backbone atoms 
(i.e., $\mathrm{C} \alpha, \mathrm{N}, \mathrm{C}$ and $\mathrm{O}$ ) of all amino acid residues in thrombin. For each system, RMSD values at a time point were averaged over $100 \mathrm{MD}$ trajectories. Assuming the convergence of RMSD (Figure S3), we analyzed trajectories in period between 20 and $25 \mathrm{~ns}$.

Hydrogen bond (HB) formation between Asp189 and ArgP1 was analyzed by using the cpptraj module in AmberTools 1.5 package. ${ }^{5}$ The criterion of $\mathrm{HB}$ formation is the same as that discussed in the Materials and Methods. The number of the HB formation was calculated as mean value for each partial MD trajectory, and then these mean values were averaged over $100 \mathrm{MD}$ trajectories. As for these values, the significant difference between the systems was tested at $95 \%$ confidence interval. 


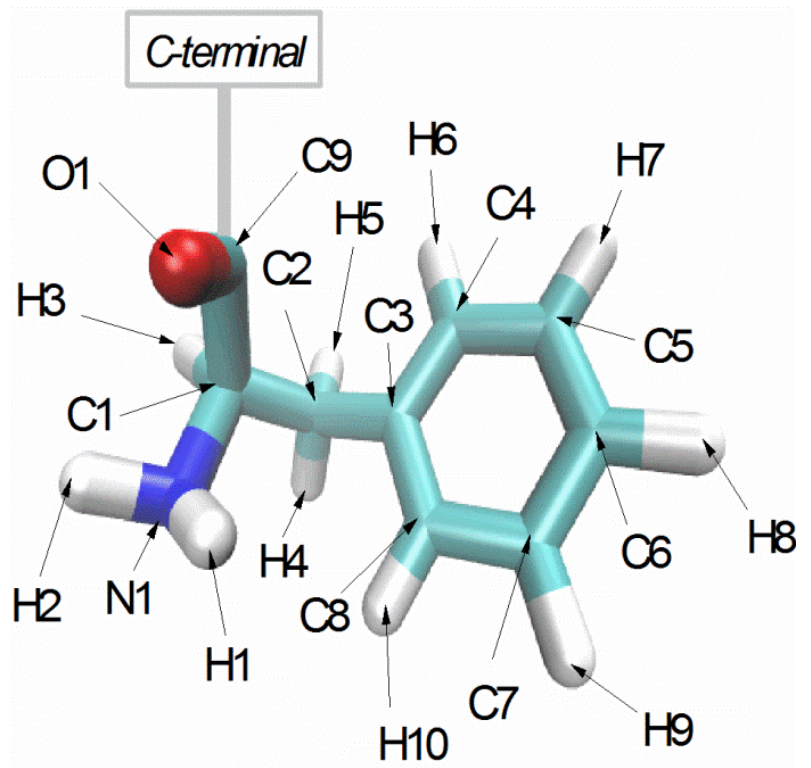

Figure S1. N-terminal phenyl alanine (DNF). 


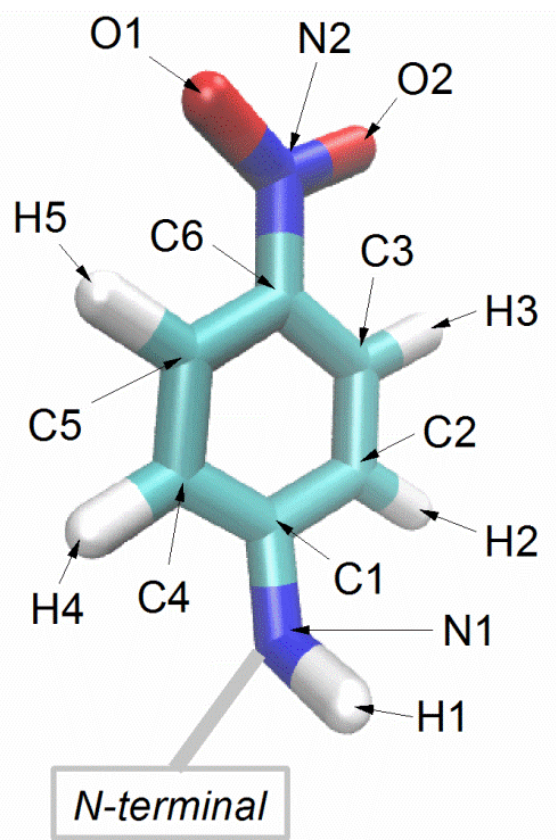

Figure S2. Para-nitroaniline (PNA). 
Table S1. Atomic charges of para-nitroaniline (PNA)

\begin{tabular}{ccc}
\hline atom name $^{a}$ & atom type $^{a}$ & RESP charge [a.u.] \\
\hline N1 & $\mathrm{n}$ & -0.606162 \\
H1 & hn & 0.310627 \\
C1 & ca & 0.377643 \\
C2 & ca & -0.238034 \\
H2 & ha & 0.190277 \\
C3 & ca & -0.169862 \\
H3 & ha & 0.202657 \\
C4 & ca & -0.238034 \\
H4 & ha & 0.190277 \\
C5 & ca & -0.169862 \\
H5 & ha & 0.202657 \\
C6 & ca & 0.031776 \\
N2 & no & 0.823390 \\
O1 & o & -0.453675 \\
O2 & o & -0.453675 \\
\hline \multicolumn{4}{c}{ total } & & 0.000000 \\
\hline
\end{tabular}

${ }^{a}$ Atom name and type follow the style of GAFF. ${ }^{14,15}$ 
Table S2. Atomic charges of N-terminal phenyl alanine (DNF)

\begin{tabular}{|c|c|c|}
\hline atom name $^{a}$ & atom type $^{a}$ & RESP charge [a.u.] \\
\hline N1 & n3 & -0.928706 \\
\hline H1 & hn & 0.342272 \\
\hline $\mathrm{H} 2$ & hn & 0.342272 \\
\hline $\mathrm{C} 1$ & c3 & 0.137960 \\
\hline $\mathrm{H} 3$ & h1 & 0.122216 \\
\hline $\mathrm{C} 2$ & $\mathrm{c} 3$ & -0.365978 \\
\hline $\mathrm{H} 4$ & hc & 0.129312 \\
\hline H5 & hc & 0.129312 \\
\hline $\mathrm{C} 3$ & $\mathrm{ca}$ & 0.192206 \\
\hline $\mathrm{C} 4$ & $\mathrm{ca}$ & -0.178917 \\
\hline H6 & ha & 0.154622 \\
\hline $\mathrm{C} 5$ & $\mathrm{ca}$ & -0.175894 \\
\hline $\mathrm{H} 7$ & ha & 0.144880 \\
\hline C6 & $\mathrm{ca}$ & -0.121475 \\
\hline $\mathrm{H} 8$ & ha & 0.133876 \\
\hline $\mathrm{C} 7$ & $\mathrm{ca}$ & -0.175894 \\
\hline H9 & ha & 0.144880 \\
\hline $\mathrm{C} 8$ & $\mathrm{ca}$ & -0.178917 \\
\hline H10 & ha & 0.154622 \\
\hline C9 & $\mathrm{c}$ & 0.585156 \\
\hline $\mathrm{O} 1$ & o & -0.587805 \\
\hline \multicolumn{2}{|c|}{ total } & 0.000000 \\
\hline
\end{tabular}

${ }^{a}$ Atom name and type follow the style of GAFF. ${ }^{14,15}$ 

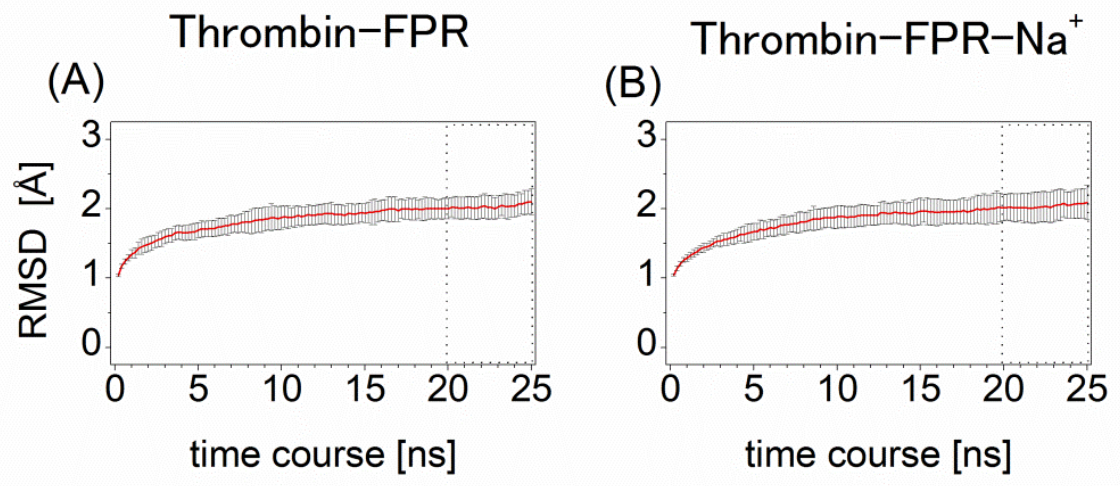

Figure S3. Ensemble average of RMSD at each time point. (A) Thrombin-FPR complex system. (B) Thrombin-FPR-Na ${ }^{+}$complex system. Error bars denote $95 \%$ confidential interval. 


\section{SI-2. Representative MD trajectories undergoing transient hydrogen}

bond formation and breakage

Panels (A) and (B) in Figure S4 illustrate $\mathrm{HB}^{\mathrm{P} 1-189}$ formation for representative $\mathrm{MD}$ trajectories of apo and holo systems, respectively. In both of the panels, we can find transient $\mathrm{HB}^{\mathrm{P} 1-189}$ formation during the period between 0 and $\tau^{\mathrm{HB}}$ ns. In the panel (B), transient $\mathrm{HB}^{\mathrm{P} 1-189}$ breakage is observed around $13.5 \mathrm{~ns}$, the time point after $\tau^{\mathrm{HB}}$. 
(A)

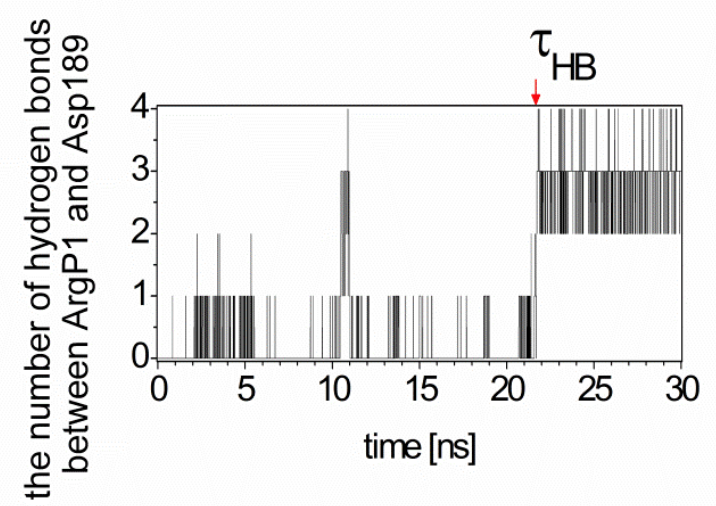

(B)

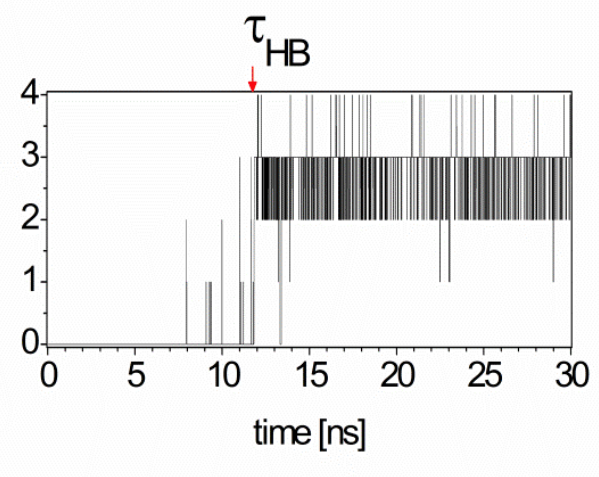

Figure S4. ArgP1-Asp189 hydrogen bond $\left(\mathrm{HB}^{\mathrm{Pl} 1189}\right)$ formation for representative trajectories. (A) apo system. (B) holo system. $\tau^{\mathrm{HB}}$ is the starting point of stable $\mathrm{HB}^{\mathrm{Pl} 189}$ formation, defined in Materials and Methods. 
SI-3. Local network of water molecules between the bound $\mathrm{Na}^{+}$and Asp189

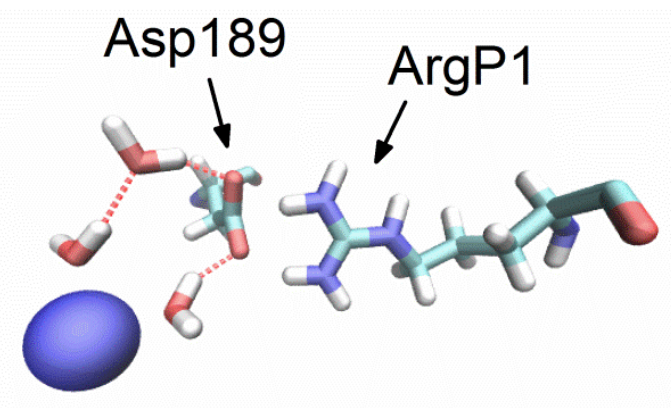

Figure S5. Representative illustration for local network of water molecules between the bound $\mathrm{Na}^{+}$and Asp189. The bound $\mathrm{Na}^{+}$is colored in blue. Hydrogen bonds between Asp189 and water molecules are shown by red dotted line. 


\section{SI-4. Time course profiles of accumulated works}

The panel A in Figure S6 illustrates the time course profiles of work for $\mathrm{HB}^{\mathrm{P} 1-189}$ formation. The profiles of apo and holo systems have an initial rises around 0.1 and 0.13 ns, respectively. The faster rise of holo system's profile suggests the influence of interaction between $\mathrm{ArgP} 1$ and the bound $\mathrm{Na}^{+}$. During the period between 0.38 and 0.45 ns, the difference in work between the two systems increases. Both of the two systems assume similar distance value at $0.45 \mathrm{~ns}$ again. This observation indicates that the repulsive interaction between $\mathrm{ArgP} 1$ and the bound $\mathrm{Na}^{+}$prevents the substrate access to S1-pocket. Since the number of $\mathrm{HB}^{\mathrm{P} 1-189}$ reaches the threshold value 2.8 by the end of SMD simulations (panel $\mathrm{B}$ in Figure S6), we assumed that the $\mathrm{HB}^{\mathrm{P} 1-189}$ formation converges during $0.5 \mathrm{~ns}$.

The panel A in Figure S7 illustrates the time course profiles of work for $\mathrm{HB}^{\mathrm{P} 1-189}$ breakage. The profiles of apo and holo systems have an initial rises at $0.07 \mathrm{~ns}$. Meanwhile, that of holo system starts to leave from that of apo system around $0.25 \mathrm{~ns}$ and, hereafter, the difference between the two systems increases. $\mathrm{HB}^{\mathrm{P} 1-189}$ breakage completes around $0.4 \mathrm{~ns}$ (panel B in Figure S7). Since holo system shows relatively rapid change in the accumulated work compared with apo system, it could be supposed that repulsive interaction between $\mathrm{ArgP} 1$ and the bound $\mathrm{Na}^{+}$promotes $\mathrm{HB}^{\mathrm{P} 1-189}$ 
breakage.

The accumulated works at $0.5 \mathrm{~ns}$ for $\mathrm{HB}^{\mathrm{P} 1-189}$ formation, ca. $19 \mathrm{kcal} / \mathrm{mol}$, is larger than those for $\mathrm{HB}^{\mathrm{P} 1-189}$ breakage, ca. $16 \mathrm{kcal} / \mathrm{mol}$. This is simply due to difference in the procedure of SMD simulations. The distance between $\mathrm{C} \zeta^{\mathrm{ArgP} 1}$ and $\mathrm{C} \gamma^{\mathrm{Asp} 189}$ changes by $6.1 \AA$ and $3.2 \AA$ in $\mathrm{HB}^{\mathrm{P} 1-189}$ formation and breakage SMD simulations, respectively (Panels $\mathrm{C}$ in Figures S6 and S7). The longer distance change results in the larger accumulated work. 


\section{apo (without $\mathrm{Na}^{+}$)}

(A)

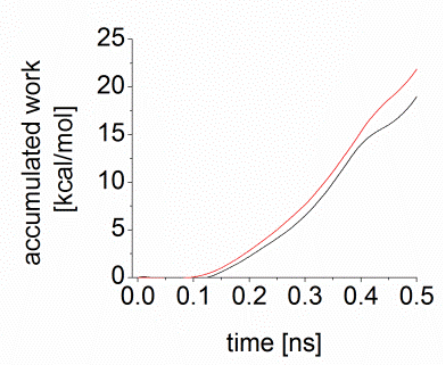

(B)

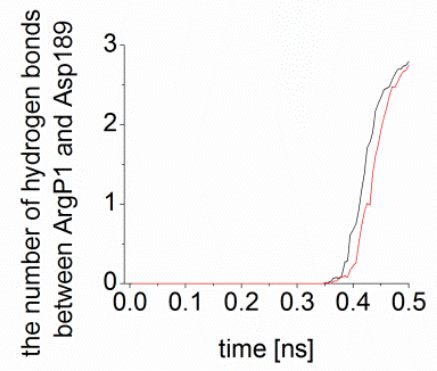

(C)

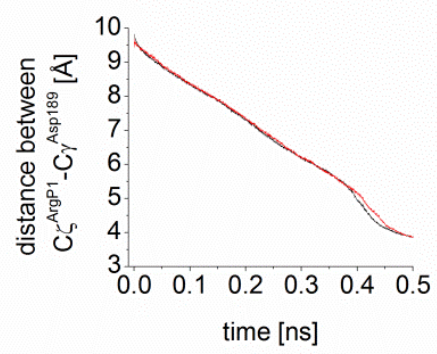

Figure S6. Time course analyses of SMD for $\mathrm{HB}^{\mathrm{P} 1-189}$ formation. (A) Accumulated work. (B) The number of $\mathrm{HB}^{\mathrm{P} 1-189}$. (C) Interatomic distance between $\mathrm{C} \zeta^{\mathrm{ArgP} 1}$ and $\mathrm{C} \gamma^{\text {Asp189. }}$. A value in each time point is an average over 96 independent SMD trajectories. 


\section{apo (without $\mathrm{Na}^{+}$) holo (with $\mathrm{Na}^{+}$)}

(A)

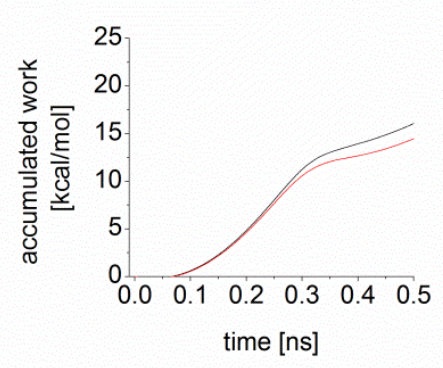

(B)

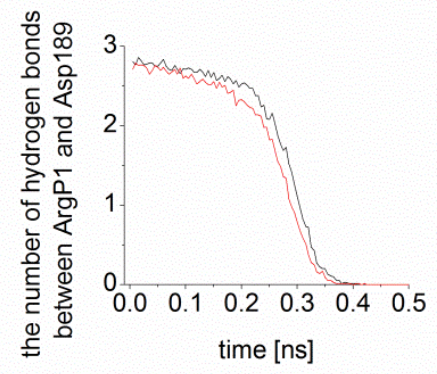

(C)

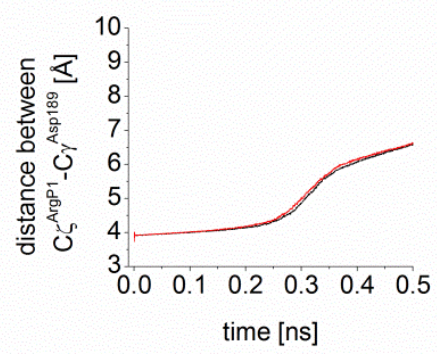

Figure S7. Time course analyses of SMD for $\mathrm{HB}^{\mathrm{P} 1-189}$ breakage. (A) Accumulated work.

(B) The number of $\mathrm{HB}^{\mathrm{P} 1-189}$. (C) Interatomic distance between $\mathrm{C} \zeta^{\mathrm{ArgP} 1}$ and $\mathrm{C} \gamma^{\mathrm{Asp} 189}$. A value in each time point is an average over 192 independent SMD trajectories. 


\section{References}

(1) Bode, W.; Mayr, I.; Baumann, U.; Huber, R.; Stone, S. R.; Hofsteenge, J. The

Refined 1.9 a Crystal-structure of Human Alpha-thrombin-interaction with D-Phe-Pro-Arg Chloromethylketone and Significance of the Tyr-Pro-Pro-Trp Insertion Segment. EMBO J. 1989, 8, 3467-3475.

(2) Di Cera, E.; Dang, Q. D.; Ayala, Y. M. Molecular Mechanisms of Thrombin Function. Cell Mol. Life Sci. 1997, 53, 701-730.

(3) Pineda, A. O.; Carrell, C. J.; Bush, L. A.; Prasad, S.; Caccia, S.; Chen, Z. W.; Mathews, F. S.; Di Cera, E. Molecular Dissection of $\mathrm{Na}^{+}$Binding to Thrombin. J. Biol. Chem. 2004, 279, 31842-31853.

(4) Pettersen, E. F.; Goddard, T. D.; Huang, C. C.; Couch, G. S.; Greenblatt, D. M.; Meng, E. C.; Ferrin, T. E. UCSF chimera - A visualization system for exploratory research and analysis. J. Comput. Chem. 2004, 25, 1605-1612.

(5) Case, D. A.; Darden, T. A.; T.E. Cheatham, I.; Simmerling, C. L.; Wang, J.; Duke, R. E.; Luo, R.; Walker, R. C.; Zhang, W.; Merz, K. M. et al. AmberTools 1.5; University of California, San Francisco, CA, 2010.

(6) Hornak, V.; Abel, R.; Okur, A.; Strockbine, B.; Roitberg, A.; Simmerling, C. Comparison of Multiple Amber Force Fields and Development of Improved Protein Backbone Parameters. Proteins 2006, 65, 712-725. 
(7) Jorgensen, W. L.; Chandrasekhar, J.; Madura, J. D.; Impey, R. W.; Klein, M. L. Comparison of Simple Potential Functions for Simulating Liquid Water. J. Chem. Phys. 1983, 79, 926-935.

(8) Kusalik, P. G.; Svishchev, I. M. The Spatial Structure in Liquid Water. Science 1994, 265, 1219-1221.

(9) Joung, I. S.; Cheatham, T. E. Determination of Alkali and Halide Monovalent Ion Parameters for Use in Explicitly Solvated Biomolecular Simulations. J. Phys. Chem. B 2008, 112, 9020-9041.

(10) Joung, I. S.; Cheatham, T. E., III Molecular Dynamics Simulations of the Dynamic and Energetic Properties of Alkali and Halide Ions Using Water-Model-Specific Ion Parameters. J. Phys. Chem. B 2009, 113, 13279-13290.

(11) Doig, A. J.; Baldwin, R. L. N- and C-Capping Preferences for All 20 Amino-Acids in Alpha-Helical Peptides. Protein Sci. 1995, 4, 1325-1336.

(12) Suenaga, N. Facio; Kyusyu University, Japan, 2012.

(13) Frisch, M. J.; Trucks, G. W.; Schlegel, H. B.; Scuseria, G. E.; Rob, M. A.; Cheeseman, J. R.; Jr., J. A. M.; Vreven, T.; Kudin, K. N.; Burant, J. C.et al.; Gaussian03; Gaussian, Inc.: Wallingford, 2003.

(14) Wang, J. M.; Wolf, R. M.; Caldwell, J. W.; Kollman, P. A.; Case, D. A. 
Development and Testing of a General Amber Force Field. J. Comput. Chem. 2004, 25, $1157-1174$

(15) Wang, J. M.; Wang, W.; Kollman, P. A.; Case, D. A. Automatic Atom Type and Bond Type Perception in Molecular Mechanical Calculations. J. Mol. Graphics Modell. 2006, 25, 247-260.

(16) Kurisaki, I.; Takayanagi, M.; Nagaoka, M. Toward Understanding Allosteric Activation of Thrombin: A Conjecture for Important Roles of Unbound $\mathrm{Na}^{+}$Molecules around Thrombin. J. Phys. Chem. B 2015, 119, 3635-3642.

(17) Berendsen, H. J. C.; Postma, J. P. M.; Vangunsteren, W. F.; Dinola, A.; Haak, J. R. Molecular-dynamics with Coupling to an External Bath. J. Chem. Phys. 1984, 81, $3684-3690$

(18) Case, D. A.; Darden, T. A.; T.E. Cheatham, I.; Simmerling, C. L.; Wang, J.; Duke, R. E.; Luo, R.; Walker, R. C.; Zhang, W.; Merz, K. M.et al. Amber12; University of California, San Francisco, CA, 2012.

(19) Le Grand, S.; Gotz, A. W.; Walker, R. C. SPFP: Speed without Compromise-A Mixed Precision Model for GPU Model Molecular Dynamics Simulations. Comput. Phys. Commun. 2013, 184, 374-380. 